\title{
NOUVELle
}

\section{Stress au travail et usage chronique de benzodiazépines}

\section{Résultats prospectifs issus de la cohorte CONSTANCES}

Guillaume Airagnes
DMU de psychiatrie et addictologie, AP-HP. Centre-université de Paris. Cohortes épidémiologiques en population, Inserm UMS 011.

Hôpital Européen Georges Pompidou, 20 rue Leblanc, 75015 Paris, France.

guillaume.airagnes@aphp.fr
> Les benzodiazépines sont les psychotropes les plus prescrits au monde, principalement en raison de leurs propriétés anxiolytiques [1]. Malgré des indications limitées, elles sont fréquemment prescrites en dehors des recommandations de bonnes pratiques qui stipulent de ne pas dépasser 12 semaines de traitement [2]. Leur usage chronique au-delà de cette durée est en effet associé à des risques augmentés d'effets indésirables comprenant, à court terme, les risques de chutes et d'accidents, et à plus long terme, les risques de dépendance et de troubles cognitifs. L'usage chronique de benzodiazépines a également été associé à un risque augmenté de maladie d'Alzheimer et de certains cancers [1].

Dans la population française, l'usage chronique de benzodiazépines concernerait $2,8 \%$ des hommes et $3,8 \%$ des femmes [2]. Les sujets les plus vulnérables aux effets indésirables de ces molécules sont paradoxalement ceux qui sont le plus à risque d'en faire un usage chronique, par exemple les sujets de plus de 50 ans ou ceux qui présentent un état dépressif [2]. Le risque d'usage chronique de benzodiazépines est également accru lorsque les conditions sociodémographiques sont défavorables. Par ailleurs, l'exposition aux risques psychosociaux, qui concerne environ un travailleur sur quatre [3], pourrait être associée à un risque augmenté d'usage chronique de benzodiazépines, au moins en cas d'exposition professionnelle stressante à un public [4]. Alors que des associations longitudinales ont déjà été décrites entre le stress au travail et les usages d'alcool, de tabac et de cannabis, le lien entre le stress au travail et l'usage chronique de benzodiazépines a été peu étudié. L'objectif de cette étude était donc de rechercher des associations prospectives entre le stress au travail et l'usage chronique de benzodiazépines, en tenant compte des facteurs sociodémographiques et des comorbidités (trouble de l'usage de l'alcool, tabagisme, dépression, et altération de l'état de santé général) [5]. Les résultats pourraient déboucher sur des actions d'information et de prévention sur les risques liés à l'existence d'un stress au travail dans le cadre de la santé publique et de la santé au travail.

La population de l'étude était composée de 31077 sujets inclus dans la cohorte CONSTANCES entre 2012 et 2014 (www. constances.fr). Ces sujets étaient tous en activité au moment de leur recrutement dans cette cohorte, et n'avaient pas présenté d'usage chronique de benzodiazépines durant les deux années précédant l'inclusion. L'usage chronique de benzodiazépines était défini comme un usage continu pendant au moins 12 semaines, en se fondant sur les données objectives de consommation de soins issues du système national d'information inter-régimes de l'assurance maladie (SNIIRAM). Le stress au travail était mesuré à l'aide d'une échelle d'évaluation standardisée du déséquilibre effort-récompense, utilisée en quartiles [6]. Les odds ratios d'usage chronique de benzodiazépines au cours d'un suivi de deux ans et en fonction du stress au travail étaient calculés par régression logistiquel. Les analyses ont d'abord été ajustées pour l'âge, le genre, le niveau d'éducation, la catégorie professionnelle, les revenus du foyer, le statut marital, le statut tabagique, et la présence éventuelle du «trouble de l'usage de l'alcool » en utilisant l'alcohol use disorder identification test. Des ajustements supplémentaires ont ensuite été effectués pour l'état de santé perçu et la présence éventuelle de signes de dépression en utilisant l'échelle CES-D (center for epidemiologic studies-depression). À l'issue des deux années de suivi, 294 participants $(0,9 \%)$ ont présenté un usage chronique de benzodiazépines. Le stress au travail mesuré à l'inclusion dans la cohorte CONSTANCES était associé au risque de survenue d'un usage chronique de benzodiazépines avec des odds ratios de 1,95 (intervalle de confiance à $95 \%: 1,32-2,87$ ) et de 2,80 (IC $95 \%: 1,96-4,01$ ) respectivement pour les troisième et quatrième quartiles comparés au premier. Aucune interaction n'était observée entre le stress au travail et les autres variables analysées, à l'exception du statut tabagique (odds ratio $=1,89$; IC $95 \%$ : 1,05-3,41; $p=0,03$ ). Les odds ratios étaient, chez les fumeurs, respectivement de 2,68 (IC $95 \%$ : 1,17-

\footnotetext{
${ }^{1}$ La régression logistique, l'un des modèles d'analyse multivariée les plus couramment utilisés en épidémiologie, est un modèle de régression binomiale. Elle permet de mesurer l'association entre la survenue d'un évènement (variable expliquée qualitative) et les facteurs susceptibles de l'influencer (variables explicatives). Le choix des variables explicatives intégrées au modèle de régression logistique est fondé sur une connaissance préalable de la physiopathologie de la maladie et sur l'association statistique entre la variable et l'évènement, mesurée par l'odds ratio (également appelé risque relatif rapproché).
} 


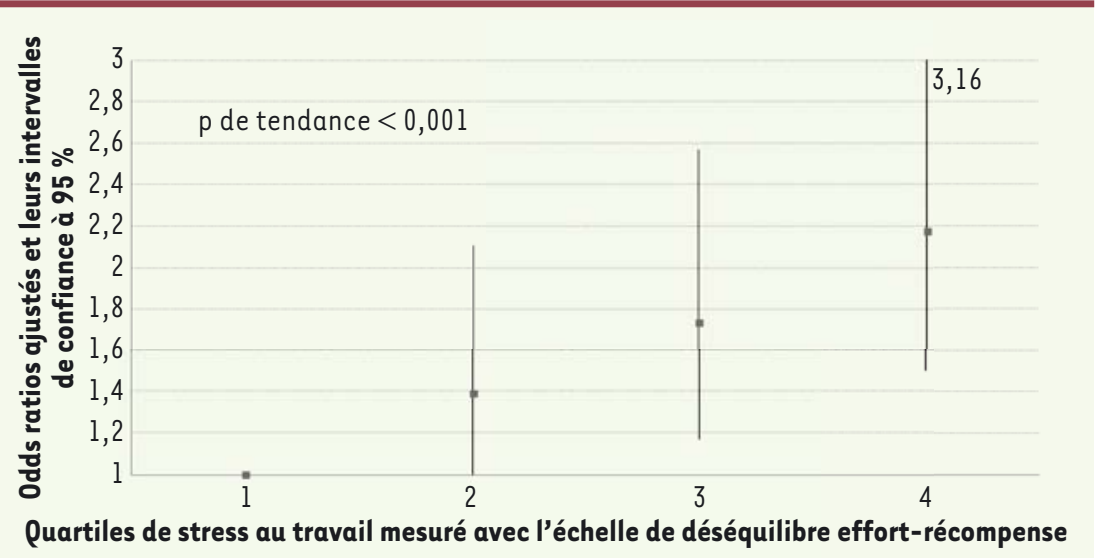

Figure 1. Associations entre le stress au travail et l'incidence d'usage chronique de benzodiazépines ajustées pour l'âge, le genre, le niveau d'éducation, la catégorie professionnelle, les revenus du foyer, le statut marital, le statut tabagique, le trouble de l'usage de l'alcool, l'état dépressif et l'état de santé général.

$6,13$ ) et de 4,74 (IC $95 \%: 2,31-9,72)$ pour les troisième et quatrième quartiles comparés au premier, tandis qu'ils étaient respectivement de 1,70 (IC $95 \%: 1,07-2,69$ ) et de 2,16 (IC $95 \%$ : $1,41-3,30)$ chez les non-fumeurs et les anciens fumeurs. Ces odds ratios étaient significativement plus élevés chez les fumeurs comparés aux non-fumeurs et anciens fumeurs ( $Z$-score $=2,16 ; p$ $=0,03)$. Après ajustements supplémentaires, les odds ratios étaient respectivement de 1,74 (IC $95 \%: 1,17-2,57$ ) et 2,18 (IC $95 \%: 1,50-3,16)$ pour les troisième et quatrième quartiles comparés au premier (Figure 1). Toutes les associations étaient dose-dépendantes ( $p$ de tendance $<0,001$; Figure 1 ).

À notre connaissance, il s'agit de la première étude ayant examiné prospectivement les associations entre le stress au travail et l'usage chronique de benzodiazépines dans une cohorte nationale issue de la population générale. L'usage chronique de benzodiazépines a été identifié à partir des bases de données médico-administratives de remboursement, c'est-à-dire avec une mesure objective et exhaustive des traitements délivrés. De plus, nous avions la possibilité de nous focaliser sur les «nouveaux » utilisateurs de benzodiazépines (cas incidents), en excluant ceux qui avaient présenté un usage chronique de benzodiazépines au cours des deux années précédant l'inclusion dans la cohorte. Les mesures du stress au travail, des symptômes dépressifs, et du trouble de l'usage de l'alcool reposaient sur des outils d'évaluation standardisés. Enfin, nos analyses ont tenu compte de plusieurs facteurs de confusion ${ }^{2}$ potentiels.

Toutefois, cette étude comporte plusieurs limites. Tout d'abord, le caractère prospectif de l'étude ne permet pas pour autant d'exclure la possibilité d'une causalité inverse (ou circulaire), de même que l'existence de facteurs confondants résiduels. Par exemple, parmi les facteurs potentiellement confondants qui n'ont pas été mesurés dans cette étude, la personnalité et les antécédents d'addiction pourraient expliquer à la fois la tendance au stress au travail et à l'usage chronique de benzodiazépines. Par ailleurs, il n'était pas possible de s'assurer que les médicaments délivrés étaient effectivement consommés (même s'il est peu probable qu'un individu se rende à au

\footnotetext{
${ }^{2}$ En statistique, une variable confondante, appelée aussi facteur confondant ou facteur de confusion, est une variable aléatoire qui influence à la fois la variable dépendante et les variables explicatives. Ces facteurs sont notamment à l'origine de la différence entre corrélation et causalité.
}

moins quatre reprises à la pharmacie pour se faire délivrer un traitement sur ordonnance sans que ce soit pour le consommer) et la méthode d'identification de l'usage de benzodiazépines ne tenait pas compte des consommations sans prescription (e.g., consommation de benzodiazépines prescrites pour quelqu'un d'autre, achat de benzodiazépines au marché noir).

$\varepsilon$ n conclusion, cette étude a mis en évidence l'association entre le stress au travail et le risque d'usage chronique de benzodiazépines au cours d'un suivi de deux ans, en tenant compte des facteurs sociodémographiques, du statut tabagique, de la présence éventuelle du trouble de l'usage de l'alcool ou d'une dépression, et de l'état de santé perçu. En situation de stress au travail, l'usage de benzodiazépines pourrait être favorisé par la volonté de réduire ce stress ou ses conséquences, et d'améliorer ses performances au travail [7]. L'exposition chronique au stress ainsi que les effets addictogènes des benzodiazépines pourraient alors en favoriser l'usage chronique. Les risques plus élevés chez les fumeurs sont en accord avec une plus grande sensibilité des fumeurs aux situations stressantes [8]. De plus, les fumeurs sont plus enclins à utiliser des substances pour faire face au stress, et il existe vraisemblablement un profil de vulnérabilité commun à l'usage des benzodiazépines et à celui du tabac en situation de stress. Quoi qu'il en soit, le repérage et la prévention du risque d'usage chronique de benzodiazépines devraient être systématisés chez les personnes qui sont en situation de stress au travail, y compris chez celles qui n'ont pas d'autres indicateurs de vulnérabilité. II existe des échelles standardisées d'évaluation de la dépendance aux benzodiazépines, telles que BenDep-SRQ [9], et la prise en charge thérapeutique peut s'appuyer sur des recommandations internationales [10]. $\diamond$

Job stress and benzodiazepines long-term use: prospective findings from the CONSTANCES cohort 


\section{LIENS D'INTÉRÊT}

L'auteur déclare avoir reçu des honoraires comme orateur et/ou consultant de Lundbeck, Pfizer et Pierre Fabre.

\section{RÉFÉRENCES}

1. Airagnes G, Pelissolo A, Lavallee M, et al. Benzodiazepine misuse in the elderly: risk factors, consequences, and management. Curr Psychiatry Rep $2016 ; 18: 89$

2. Airagnes G, Lemogne C, Renuy A, et al. Prevalence of prescribed benzodiazepine long-term use in the French general population according to sociodemographic and clinical factors: findings from the CONSTANCES cohort. BMC Public Health $2019 ; 19$ : 566.
3. Bègue $C$, Fouquet N, Bodin J, et al. Evolution of psychosocial factors at work in a French region. Occup Med $2015 ; 66: 128-34$

4. Airagnes $G$, Lemogne $C$, Olekhnovitch $R$, et al. Workrelated stressors and increased risk of benzodiazepine long-term use: findings from the CONSTANCES populationbased cohort. AmJPublic Health 2019 ; 109 : 119-25.

5. Airagnes G, Lemogne C, Kab S, et al. Effort-reward imbalance and long-term benzodiazepine use: longitudinal findings from the CONSTANCES cohort. J Epidemiol Community Health 2019 ; 73 : 993-1001.

6. Siegrist J, Wahrendorf M, Goldberg $M$, et al. Is effortreward imbalance at work associated with different domains of health functioning? Baseline results from the French CONSTANCES study. Int Arch Occup Environ Health $2018 ; 92: 467$.

7. Ngoundo-Mbongue TB, Niezborala M, Sulem P, et al. Psychoactive drug consumption: performance-

\section{NOUVELLE}

\section{Comment le stress précoce altère le comportement à l'âge adulte}

Anne Teissier, Patricia Gaspar enhancing behaviour and pharmacodependence in workers. Pharmacoepidemiol Drug Saf 2005 ; 14 : 81-9.

8. Back SE, Waldrop AE, Saladin ME, et al. Effects of gender and cigarette smoking on reactivity to psychological and pharmacological stress provocation. Psychoneuroendocrinol $2008 ; 33: 560$ 8.

9. Kan CC, van der Ven AH, Breteler MH, Zitman FG. Latent trait standardization of the benzodiazepine dependence self-report questionnaire using the Rasch scaling model. Compr Psychiatry $2001 ; 42$ : 424-32.

10. Soyka M. Treatment of benzodiazepine dependence. N Engl J Med 2017 ; 376 : 1147-57.
> L'environnement dans lequel un enfant grandit joue un rôle crucial dans le développement de son cerveau et l'établissement d'un équilibre psychique. On sait, grâce à de nombreuses études épidémiologiques, que l'exposition à des traumatismes pendant l'enfance (notamment par négligence émotionnelle ou physique, ou par des abus émotionnels, physiques, ou sexuels) augmente le risque de développer des troubles psychiques tels qu'un état anxieux ou dépressif, et constitue un facteur aggravant pour des maladies psychiatriques comme la bipolarité ou la schizophrénie [1]. On estime que $30 \%$ du risque de maladie psychiatrique est imputable aux traumatismes de l'enfance [2], ce qui fait de leur prévention un enjeu important de santé publique.

Les mécanismes biologiques impliqués restent mal compris. L'étude des cerveaux de sujets adultes ayant été exposés à un stress pendant l'enfance a permis d'identifier des altérations de l'activité corticale et de la substance blanche, notamment dans le cortex préfrontal, une région cérébrale impliquée dans le contrôle émotionnel et la cognition [3]. Pour tenter de comprendre l'origine des anomalies comportementales persistantes après un stress précoce, nous avons entrepris d'identifier les mécanismes moléculaires et cellulaires qui sous-tendent la perturbation du développement cérébral associée.

\section{Les étapes d'un développement cérébral normal}

Les neurones sont essentiellement générés pendant la vie embryonnaire. Puis, durant une période de maturation, ils étendent leurs axones et leurs dendrites, avant d'émettre des potentiels d'action et d'établir des contacts synaptiques afin de former des réseaux fonctionnels. En parallèle de ce processus de maturation neuronale a lieu la gliogenèse, c'est-àdire la production des cellules gliales (astrocytes, oligodendrocytes, et cel-
Institut du Fer à Moulin, Inserm UMRS 839 / Sorbonne Université, Paris, France.

Institut de Psychiatrie et Neurosciences de Paris, Inserm UMRS 1266, Paris, France. Institut du Cerveau et de la Moelle épinière, CHU Pitié-Salpêtrière, 75013 Paris, France. anne.teissier@inserm.fr patricia.gaspar@inserm.fr

lules microgliales) et leur différenciation. Les cellules gliales sont essentielles au fonctionnement cérébral $[4](\rightarrow)$ : $\rightarrow$ Voir l'éditorial d'Y. Agid et X. Fan, $\mathrm{m} / \mathrm{s} \mathrm{n}^{\circ} 3$, mars 2019 , page 199 les astrocytes subviennent aux besoins énergétiques des neurones, les cellules microgliales contrôlent la maturation synaptique, tandis que les oligodendrocytes synthétisent la gaine de myéline autour des axones, ce qui permet une conduction plus rapide de l'influx nerveux. Ces phénomènes de maturation neuronale et de gliogenèse, qui débutent durant la vie intra-utérine et se prolongent dans l'enfance, sont donc les plus susceptibles d'être affectés par un stress précoce. Plus tard, durant l'adolescence, une étape de sélection des connexions neuronales qui persisteront dans le cerveau adulte permet une optimisation des réseaux neuronaux et ainsi l'adaptation à l'environnement. Les processus moléculaires contrôlant le développement cérébral étant pour la 